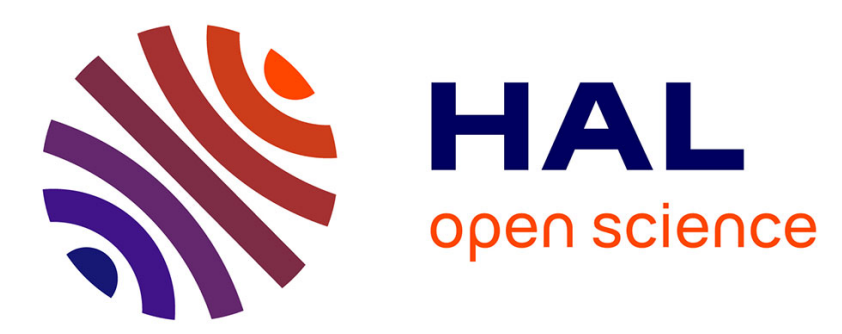

\title{
Hopping conductivity of Ni-doped p-CdSb in strong magnetic fields
}

\author{
R. Laiho, A.V. Lashkul, K.G. Lisunov, E. Lähderanta, M.A. Shakhov, V.S. \\ Zakhvalinskii
}

\section{- To cite this version:}

R. Laiho, A.V. Lashkul, K.G. Lisunov, E. Lähderanta, M.A. Shakhov, et al.. Hopping conductivity of Ni-doped p-CdSb in strong magnetic fields. Journal of Physics and Chemistry of Solids, 2009, 70 (2), pp.428. 10.1016/j.jpcs.2008.11.015 . hal-00508474

\section{HAL Id: hal-00508474 https://hal.science/hal-00508474}

Submitted on 4 Aug 2010

HAL is a multi-disciplinary open access archive for the deposit and dissemination of scientific research documents, whether they are published or not. The documents may come from teaching and research institutions in France or abroad, or from public or private research centers.
L'archive ouverte pluridisciplinaire HAL, est destinée au dépôt et à la diffusion de documents scientifiques de niveau recherche, publiés ou non, émanant des établissements d'enseignement et de recherche français ou étrangers, des laboratoires publics ou privés. 


\section{Author's Accepted Manuscript}

Hopping conductivity of Ni-doped p-CdSb in strong magnetic fields

R. Laiho, A.V.Lashkul, K.G. Lisunov, E. Lähderanta, M.A. Shakhov, V.S. Zakhvalinskii

PII: $\quad$ S0022-3697(08)00542-8

DOI: $\quad$ doi:10.1016/j.jpcs.2008.11.015

Reference: $\quad$ PCS 5757

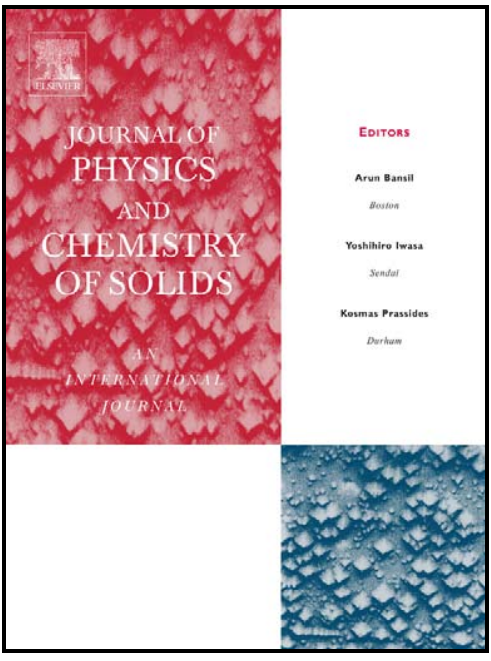

www.elsevier.com/locate/jpcs

To appear in: Journal of Physics and Chemistry of Solids

Received date: 8 April 2008

Revised date: $\quad 4$ November 2008

Accepted date: 19 November 2008

Cite this article as: R. Laiho, A.V. Lashkul, K.G. Lisunov, E. Lähderanta, M.A. Shakhov and V.S. Zakhvalinskii, Hopping conductivity of Ni-doped p-CdSb in strong magnetic fields, Journal of Physics and Chemistry of Solids (2008), doi:10.1016/j.jpcs.2008.11.015

This is a PDF file of an unedited manuscript that has been accepted for publication. As a service to our customers we are providing this early version of the manuscript. The manuscript will undergo copyediting, typesetting, and review of the resulting galley proof before it is published in its final citable form. Please note that during the production process errors may be discovered which could affect the content, and all legal disclaimers that apply to the journal pertain. 


\section{Hopping conductivity of Ni-doped p-CdSb in strong magnetic fields}

R. Laiho ${ }^{1}$, A. V. Lashkul ${ }^{1,2}$, K. G. Lisunov ${ }^{1,2,3}$, E. Lähderanta ${ }^{2}$, M. A. Shakhov ${ }^{1,4}$, and V. S. Zakhvalinskii $^{1,5}$

${ }^{1}$ Wihuri Physical Laboratory, University of Turku, FIN-20014 Turku, Finland

${ }^{2}$ Department of Physics and Mathematics, Lappeenranta University of Technology, FIN-53851 Lappeenranta, Finland

${ }^{3}$ Institute of Applied Physics, Academiei Str. 5, MD-2028 Kishinev, Moldova

${ }^{4}$ A. F. Ioffe Physico-Technical Institute, 194021 St. Petersburg, Russia

${ }^{5}$ Department of Physics, Belgorod State University, RUS-308015 Belgorod, Russia

Magnetoresistance (MR) of oriented single crystals of the anisotropic semiconductor $p$-CdSb doped with 2 at $\%$ of $\mathrm{Ni}$ is investigated between $T=1.5-300 \mathrm{~K}$ in transversal pulsed magnetic fields up to $B=30 \mathrm{~T}$. In fields $B \sim 4-15 \mathrm{~T}$ at $T$ below $4.2 \mathrm{~K}$ the resistivity obeys the law $\ln \rho \sim \eta[B \varphi$ $(B)]^{1 / 2}$ with $\varphi(B)=a(0) / a(B)$, where $a$ is the carrier localization radius and parameter $\eta$ depends on $a(0)$, on the acceptor concentration $N_{\mathrm{A}}$ and on the direction of the magnetic field with respect to the crystallographic axes, but does not depend on $T$. Such behavior gives evidence for MR realized by hopping charge transfer over the nearest-neighbor sites in strong magnetic field. The analysis of the experimental data yields the values of $\eta$, agreeing with calculated ones within an error of $10 \%$, taking into account the effects of the anisotropy of the acceptor states and of the explicit dependence of $a(B)$ due to increase of the activation energy of shallow acceptors in magnetic field and the sensitivity of the metal-insulator transition to $B$. 


\section{INTRODUCTION}

Interest to semiconductors doped with transition-metal elements is connected to the spinpolarized electron transport, making them attractive e. g. for applications in spintronics [1]. Progress in this rapidly developing area suggests search of new diluted magnetic semiconductor systems. Recently a family of such materials has been enriched by cadmium antimonide doped with $\mathrm{Ni}(\mathrm{p}-\mathrm{CdSb}: \mathrm{Ni})$ [2]. Special interest to this system is connected to strongly anisotropic transport in the group II-V semiconductor p-CdSb [3]. In undoped material the activated conductivity is governed by acceptor bands with energies $\sim 3 \mathrm{meV}$ and $6 \mathrm{meV}$. Fe and $\mathrm{Ni}$ substituting for $\mathrm{Sb}$ in the lattice act as acceptors, whereas doping with Ag induces the metal-insulator transition (MIT) [3]. The anisotropic quantum transport revealed by Shubnikov-de Haas effect [4] and negative magnetoresistance [5] are observed on the metallic side of the MIT, whereas anisotropic hopping conductivity is realized on the insulating side of the MIT [6].

A new important feature of $\mathrm{CdSb}$ doped with $\mathrm{Ni}$ is formation of nanosize $\mathrm{Ni}$-rich $\mathrm{Ni}_{1-\mathrm{x}} \mathrm{Sb}_{\mathrm{x}}$ clusters with ferromagnetic (FM) ordering of internal Ni spins [2]. The clusters have a broad size distribution, large non-sphericity and orientations distributed along a preferred direction. The presence of these clusters leads to considerably anisotropic magnetic properties of $\mathrm{p}-\mathrm{CdSb}: \mathrm{Ni}$, as well as to frustrated ground state and spin-freezing starting at the room temperature [2], making p$\mathrm{CdSb}$ :Ni a perspective spintronics material.

Investigations of hopping transport in $\mathrm{p}-\mathrm{CdSb}: \mathrm{Ni}$ in zero and weak magnetic fields $(B<6 \mathrm{~T})$ reveal interesting behavior, uncommon of conventional (non-magnetic) semiconductors. Namely the variable-range hopping (VRH) conductivity at $B=0$ transforms into the nearest-neighbor hopping $(\mathrm{NNH})$ conductivity in nonzero field. This transition is accompanied with expansion of the temperature interval of the hopping conductivity already in weak magnetic fields, in line with presence of magnetic disorder or strongly inhomogeneous magnetization due to Ni-rich nanoclusters [7], which is damped when $B$ is increased. 
In this work detailed investigation of the hopping conductivity in $\mathrm{p}-\mathrm{CdSb}: \mathrm{Ni}$ in strong magnetic field is reported to complete the picture of hopping charge transfer in this material.

\section{EXPERIMENTAL RESULTS}

Single crystals of $\mathrm{CdSb}$ doped with 2 at $\%$ of $\mathrm{Ni}$ were prepared by the modified Bridgman method $[2,3]$. As observed by X-ray diffraction, the ingots of volume $\sim 1 \mathrm{~cm}^{3}$ were of single phase material with orthorhombic structure (space group $D_{2 h}^{15}$ ) and had the same lattice parameters as undoped $\mathrm{CdSb}$ [3]. For investigations rectangular prisms with the longest edge along the [100] (\# 1), [010] (\# 2) and [001] (\# 3) axes, respectively, were cut from the ingots. The measurements of $\rho$ (T) were made by recording the signal from two different pairs of potential contacts on the samples placed in a He exchange gas dewar, where their temperature could be varied between $1.5-300 \mathrm{~K}$ to an accuracy of $0.5 \%$. MR measurements were made in transversal field configurations with $\mathbf{j} \|$ [100] and $\mathbf{B} \|$ [001] (\# 1), j || [010] and B || [100] (\# 2), j || [001] and B || [010] (\# 3) at temperatures between $T=1.5-300 \mathrm{~K}$ in pulsed magnetic fields up to $B=30 \mathrm{~T}$. The magnetic pulse length was 8 ms, the error in the strength of the field was not larger than $5 \%$ and its inhomogeneity did not exceed $0.3 \%$.

As can be seen from Fig. 1 the values of $\rho(T)$ at $B=0$ decrease smoothly below $300 \mathrm{~K}$ until a minimum is attained at $\sim 30 \mathrm{~K}$ followed by strong increase of $\rho$ with further decreasing of $T$ [7]. As shown in Fig. 2 the data of $\rho(B)$ exhibit large positive MR, increasing with $B$ and decreasing with increasing $T$ at liquid He temperatures [approximately above the break in the $\rho(B)$ axis], whereas at higher $T$ (below this break) the dependence of $\rho$ on $B$ is weakened. In addition, MR decreases with increasing $T$ up to $\sim 30 \mathrm{~K}$ and then starts to increase, in agreement with the behavior of $\rho(T)$ at $B=0$ in Fig. 1 [7]. 


\section{ANALYSIS OF THE RESULTS AND DISCUSSION}

The plots of $\ln \rho$ vs. $T^{-1}$ in the inset to Fig. 1 exhibit two intervals of activated behavior characterized by different slopes between $\sim 5-20 \mathrm{~K}$ and below $\sim 5 \mathrm{~K}$, corresponding to the conductivity determined mainly by activation of holes from shallow acceptor states to the valence band and to the hopping conductivity over the states of the impurity band, respectively [7]. In semiconductors transversal MR, connected to non-generate carriers in conduction or valence band (VB), depends on $B$ as follows: $\rho(B)=\rho_{0}\left[1+C(\mu B)^{2}\right]$ for $\mu B<<1$ and $\rho(B)=\rho_{0}[1+D]$ for $\mu B$ $>>1$, where $\rho_{0}$ and $\mu$ are zero-field resistivity and carrier mobility, respectively, $C$ and $D$ are constants depending on a scattering mechanism [8].

Generally, the dependence of MR on $B$ in the region of conductivity over the VB states, given by these equations, is always much weaker than the exponential magnetic-field dependence of MR in the region of hopping conductivity (see below), which takes place in p-CdSb:Ni below $T \sim$ 4.2 K [7]. This explains the difference in the behavior of MR in Fig. 2 in the two temperature regions, including a strong increase with $B$ at $T \leq 4.2 \mathrm{~K}$ followed by a weaker one above $T \sim 10 \mathrm{~K}$. On the other hand, the equations above mean that temperature dependence of MR in the interval of the conductivity over the VB states is determined presumably by $\rho_{0}$ and $\mu$, both parameters being functions of $T$. In more detail this interval is presented for \# 3 (the bottom panel of Fig. 2). Namely, one can see two main features of the MR curves at $T>4.2 \mathrm{~K}$ : (i) rapid decrease of MR with decreasing $T$ between $4.2-30 \mathrm{~K}$ and slow increase in the interval of $30-300 \mathrm{~K}$; (ii) the weakening of the dependence of MR on $B$ with increasing $T$. Both these features agrees with the behavior prevised by the equations above, the feature (i) being connected to the factor $\rho(0)$ in both equations [cf. the behavior of $\rho(T)$ at $B=0$ in Fig. 1] and the feature (ii) due to decreasing $\mu(T)$ with increasing $T$, which takes place in undoped or weakly doped $\mathrm{p}$-CdSb in the corresponding temperature interval $[3,9]$.

Below we investigate MR in the low-temperature interval of hopping conductivity in more detail. 
As mentioned in the Introduction, hopping conductivity of $\mathrm{p}-\mathrm{CdSb}$ :Ni in zero and weak $(B<$ $6 \mathrm{~T})$ magnetic fields has been already investigated, yielding the data of microscopic parameters such as the mean localization radius, $a \equiv a(0)$, the acceptor concentration, $N_{\mathrm{A}}$, and the anisotropy coefficients $p_{\mathrm{j}}=\left[m_{\mathrm{j}}^{2} /\left(m_{\mathrm{k}} m_{\mathrm{l}}\right)\right]^{1 / 6}$, where $m_{\mathrm{j}}, m_{\mathrm{k}}$ and $m_{\mathrm{l}}$ are the components of the hole effective mass. The subscripts $j, k, l=1,2,3(j \neq k \neq l)$ and $j=3,1,2$ for \# 1, \# 2 and \# 3, respectively, correspond to the direction of the magnetic field along the [001], [100] and [010] axes, respectively (see Table I) [7]. As follows from Table I, the relation $a>a_{0}$, corresponds to proximity to MIT of the acceptor system in all investigated samples (where $a_{0}=83.5 \AA$ is the value of $a$ far from the MIT) and the values of $N_{\mathrm{A}}$ are relatively close to the critical MIT acceptor concentration $N_{\mathrm{C}}=6.28 \times 10^{16} \mathrm{~cm}^{-3}$. In Table I are displayed also the values of the activation energy of the shallow acceptors, $E_{\mathrm{A}}$, obtained from the linear fit of the plots of $\ln \rho$ vs. $T^{-1}$ at $B=0$ in the inset to Fig. 1 [7]. In all the samples the weak-field limit corresponds to the condition of $\lambda>>a_{0}$, where $\lambda$ is the magnetic length. Under this condition the quadratic dependence of MR, $\ln \rho \sim B^{2}$, has been observed in p-CdSb:Ni [7], whereas its violation (for $\lambda \leqslant a_{0}$ ) is related to the onset of the strong-field limit of MR [10], which will be analyzed below.

As follows from the percolation model of the hopping charge transfer over the nearest sites (or NNH conductivity) [10], the positive MR is connected to shrinkage of the impurity wave functions in the direction perpendicular to $\boldsymbol{B}$ and is given in strong magnetic field by the expression $[6,10]$

$$
\ln \left[\rho(B) / \rho^{*}\right]_{\mathrm{j}}=\eta_{\mathrm{j}}[B \varphi(B)]^{1 / 2},
$$

where $\rho^{*}$ is a constant,

$$
\eta_{\mathrm{j}}=q p_{\mathrm{j}}^{1 / 2}\left[e /\left(N_{\mathrm{A}} a \hbar\right)\right]^{1 / 2}
$$

$q=0.92$ and

$$
\varphi(B)=a / a(B)
$$

Presence of the factors $p_{\mathrm{j}}$ in Eq. (2) reflects the anisotropy of the acceptor states in p-CdSb and the different elasticity of the anisotropic acceptor wave functions to magnetic shrinkage at 
different directions of $\boldsymbol{B}$ in p-CdSb $[6,10]$, which makes MR to be anisotropic and sensitive to the direction of the magnetic field via the coefficient $\eta_{j}$ in Eq. (1). On the other hand, it should be underlined that $\eta_{\mathrm{j}}$ does not depend on $T$.

Another important point of the analysis of the MR data in strong fields with Eqs. $(1-3)$ deals with origin and consecutive account of the magnetic field dependence of the localization radius, which sets in already far from the MIT, when $\lambda$ becomes comparable with $a_{0}(0) \equiv a_{0}$, and is connected to the dependence of $E_{\mathrm{A}}$ on $B[10]$. Introducing the field $B_{0} \equiv \hbar /\left(e a_{0}^{2}\right)$ with the condition of $\lambda\left(B_{0}\right)=a_{0}$, this dependence can be written as $E_{\mathrm{A}}(B)=E_{\mathrm{A}}(0) \equiv E_{\mathrm{A}}$ for $B<<B_{0}$ and $E_{\mathrm{A}}(B)=E_{\mathrm{A}}$ $\ln ^{2}\left(B / B_{0}\right)$ for $B>B_{0}[10]$. In our case of $B_{0}=9.5 \mathrm{~T}$ the interval of high fields (see below) corresponds to $B \sim B_{0}$ or $\lambda \sim a_{0}$, where $E_{\mathrm{A}}(B)$ cannot be obtained analytically [10]. On the other hand, this function can be approximated well with the relation $E_{\mathrm{A}}(B)=\beta B^{1 / 3}$, where $\beta$ is independent of $B[10]$. Although an analytical expression for $\beta$ cannot be found, it is reasonable to suppose that $\beta$ is anisotropic in $p$-CdSb. The dependence $E_{\mathrm{A}}(B)$ can be found approximately from the plots of $\ln \rho(B, T)$ vs. $T^{-1}$ in the temperature interval of the acceptor freeze-out (examples are shown for \# 1 in Fig. 3). Then $\beta$ is obtained from the plots of $E_{\mathrm{A}}$ vs. $B^{1 / 3}$ which are close to linear between $B=10-15 \mathrm{~T}$ (see the inset to Fig. 3) yielding the values collected in Table I and supporting the expected anisotropy of $\beta$. Taking into account the magnetic field dependence of $E_{\mathrm{A}}$ $(B)$ above and using the relation $a_{0} \sim E_{\mathrm{A}}^{-1 / 2}[10]$ one can find $\varphi(B)=\varphi_{0}(B)$, where $\varphi_{0}(B) \equiv a_{0} / a_{0}$ $(B)$ is given by the expression

$$
\varphi_{0}(B)=\left(\beta B^{1 / 3} / E_{\mathrm{A}}\right)^{1 / 2}
$$

On the other hand, as has been mentioned above the acceptor system in all our samples is close to the MIT. The sensitivity of MIT to $B$ brings an additional dependence of $a$ on $B$, because shrinking of the impurity wave functions by magnetic field diminishes their overlap taking the system away from the MIT at $B=0$. This process decreases $a$ when $B$ is increased. 
To take into account the dependence of MIT on $B$ we treat the acceptor band in $\mathrm{p}-\mathrm{CdSb}: \mathrm{Ni}$ as the Anderson band characterized by two mobility edges, $E_{\mathrm{c}}$ and $E_{\mathrm{c}}$ ', separating the energy interval of the delocalized states $\left(E_{\mathrm{c}}, E_{\mathrm{c}}{ }^{\prime}\right)$ and the intervals of the localized states outside it (i. e. close to the DOS tails) [11]. If $E_{\mathrm{a}}$ and $E_{\mathrm{a}}{ }^{\prime}$ are the bottom and the top of this band, respectively, and the energy is measured from $E_{\mathrm{a}}$ towards the valence band, then $E_{\mathrm{c}}{ }^{\prime}>E_{\mathrm{c}}, E_{\mathrm{a}}{ }^{\prime}>E_{\mathrm{a}}$ and $E_{\mathrm{c}}{ }^{\prime}<\mu<E_{\mathrm{a}}{ }^{\prime}$, where $\mu$ is the Fermi energy. Such position of $\mu$ corresponds to the insulating side of MIT and to the small degree of compensation, $K=N_{\mathrm{D}} / N_{\mathrm{A}}$ (where $N_{\mathrm{D}}$ is the concentration of compensating donors) in our samples [7]. In such case the relation $E_{\mathrm{a}}{ }^{\prime}-E_{\mathrm{c}}{ }^{\prime} \approx V_{0}{ }^{2} /(4 P I)$ is fulfilled, where $V_{0}$ and $P$ are constants describing the degree of disorder (the width of distribution of the hole potential energy) and the number of the nearest neighbors in the acceptor system, respectively, and $I=I\left(a_{0}, \gamma_{0}\right)=$ $I_{0}\left(a_{0}, \gamma_{0}\right) \exp \left(\gamma_{0}\right)$ is the overlap integral [11]. Here $\gamma_{0} \equiv R_{A} / a_{0}, R_{\mathrm{A}}=\left(4 \pi N_{\mathrm{A}} / 3\right)^{-1 / 3}$ is half of the mean distance between the acceptors and $I_{0}\left(\gamma_{0}, a_{0}\right)=\left(e / a_{0}\right)\left[(3 / 2)\left(1+\gamma_{0}\right)+(1 / 6) \gamma_{0}^{2}\right]$ is the prefactor for hydrogenic impurity wave functions [11]. Near MIT the localization radius can be written as $a=a_{0}$ $\left(1-E_{\mathrm{c}}{ }^{\prime} / \mu\right)^{-v}$, where $v$ is the critical exponent [11]. Taking into account that $v=1$ and the degree of compensation, $K$ is small ( $\mu$ lies close to $\left.E_{\mathrm{a}}{ }^{\prime}\right)$ [7] in the last equation we can put $\mu \approx E_{\mathrm{a}}$ and obtain $a$ $\approx a_{0} \mu\left(4 P I / V_{0}^{2}\right)$, which gives $a / a(B) \approx a_{0}(B) I(B) /\left[a_{0} I(0)\right]$, where $I(B) \equiv I\left[a_{0}(B), \gamma_{0}(B)\right]$ and $\gamma_{0}(B)$ $\equiv R_{\mathrm{A}} / a_{0}(B)$. Finally we get

$$
\varphi(B) \approx \frac{(3 / 2)\left(1+\gamma_{0}\right)+(1 / 6) \gamma_{0}^{2}}{(3 / 2)\left[1+\gamma_{0}(B)\right]+(1 / 6)\left[\gamma_{0}(B)\right]^{2}} \exp \left\{-\gamma_{0}\left[1-\varphi_{0}(B)\right]\right\}
$$

As can be seen from Fig. 4 the plots of $\ln \rho$ vs. $[B \varphi(B)]^{1 / 2}$ exhibit linear behavior in the interval of $B$ between $\sim 4-15 \mathrm{~T}$, and their slopes do not depend on temperature at $T \leq 4.2 \mathrm{~K}$ in \# 1 and \# 2 and between $3-4.2 \mathrm{~K}$ in \# 3 . The values of the slopes $\eta^{(\mathrm{ex})}$ obtained from the plots in Fig. 4 are collected in Table I. They are compared with $\eta_{j}{ }^{\text {(cal) }}$, calculated with Eq. (2) using the data of $N_{\mathrm{A}}, a$ and $p_{\mathrm{j}}$ in Table I, exhibiting deviations from them of $\sim 10 \%, 3 \%$ and $5 \%$, respectively. 
Hence, the behavior of MR in p-CdSb:Ni in the interval of $B$ between $\sim 4-15 \mathrm{~T}$ is governed by the $\mathrm{NNH}$ conductivity in the limit of strong fields, yielding a complete numerical agreement with predictions of the percolation theory of $\mathrm{NNH}$ [10], if the anisotropy of the acceptor states and explicit dependence of $a$ on $B$ are taken into account. It is worth mentioning that the good agreement between $\eta^{(\mathrm{ex})}$ and $\eta_{\mathrm{j}}^{\text {(cal) }}$ above supports the values of the parameters $N_{\mathrm{A}}, a$ and $p_{\mathrm{j}}$ obtained from investigations of the resistivity in zero and weak magnetic fields [7].

At this point, the deviations of the plots in Fig. 4 from linearity with increasing of $B$ are attributable to deviation of the function $E_{\mathrm{A}}(B)$ from the $B^{1 / 3}$ dependence expected for $B \sim B_{0}$, towards $\ln ^{2} B$ which sets in for $B>>B_{0} \sim 10 \mathrm{~T}$ [see text between Eqs. (3) and (4)], yielding weaker dependence of $a(B)$. On the other hand, the onset of the temperature dependence of $\eta_{\mathrm{j}}$ in \# 3 observed below $3 \mathrm{~K}$ (Fig. 4) cannot be attributed to transition to the VRH conductivity (as e. g. in undoped p-CdSb [6]), because for any VRH mechanism positive MR is predicted to strengthen when $T$ is decreased $[10,12]$, that is the slopes in the bottom part of Fig. 4 are expected to increase with $T$, but not vise versa. Such behavior cannot be attributed to contribution of the negative MR, connected to damping of the interference of the direct paths in conventional non-magnetic semiconductors in the regime of the VRH conductivity $[13,14]$. Indeed, the negative MR of such nature dominates in weak fields but saturates when $B$ is increased $[13,14]$, whereas no signs of the negative contribution to MR in the weak-field limit have been observed [7]. Although one cannot exclude the appearance of a contribution of the negative MR in strong fields due to presence of the magnetic Ni-rich nanoclusters in p-CdSb:Ni [2]. A final conclusion at this point requires further investigations. Hence, only the NNH conductivity can be identified unambiguously in p-CdSb:Ni in strong magnetic fields.

Finally, it is instructive to compare the analysis above with its simplified variants to discuss their applicability. The first of them is the case, sometimes met in the literature, when the magnetic field dependence of $a$ is neglected, giving $\varphi(B)=1$ and the law $\ln \left[\rho(B) / \rho^{*}\right]_{\mathrm{j}}=\eta_{\mathrm{j}} B^{1 / 2}$ instead of Eq. (1). It can be shown that the plots of $\ln \rho$ vs. $B^{1 / 2}$ also contain linear parts approximately in the same field interval of $\sim 4-15 \mathrm{~T}$ with the slopes $\left.\eta^{(e x)}\right|_{\varphi=1}$ independent of $T$ within some temperature 
intervals, which are collected in Table I, demonstrating a reasonable agreement with $\eta_{j}{ }^{(\mathrm{cal})}$ only for \# 3. The second variant of simplification is to put $\varphi(B)=\varphi_{0}(B)$ given by Eq. (4), when the sensitivity of the MIT to $B$ is neglected. It gives in a same way values of the slopes $\left.\eta^{(e x)}\right|_{\varphi=\varphi_{0}}$ displayed in Table I, which are closer to $\eta_{j}{ }^{(\text {cal })}$ for \# 1 and \# 2 than in the previous case, but again an agreement is observed only for \# 3. Hence, for two out of the three investigated samples it is important to take into account both effects leading to the dependence of $a(B)$. On the other hand, in \# 3 such dependence is weakest due to the smallest value of $\beta$ (i. e. the weakest dependence of $E_{\mathrm{A}}$ on $B$ - see Table I) and the largest difference of $N_{\mathrm{C}}-N_{\mathrm{A}}$ (the smallest proximity to the MIT - see

Table I), where $N_{\mathrm{C}}=6.28 \times 10^{16} \mathrm{~cm}^{-3}$ [7], which makes inclusion of $a(B)$ into the analysis unimportant.

\section{CONCLUSIONS}

We have investigated the resistivity and magnetoresistance of $\mathrm{p}-\mathrm{CdSb}$ with 2 at $\%$ of $\mathrm{Ni}$ between $T=1.5$ and $300 \mathrm{~K}$ in pulsed magnetic fields up to $B=30 \mathrm{~T}$. It is shown that below $4.2 \mathrm{~K}$ in the fields between $\sim 4-15 \mathrm{~T}$ the resistivity is governed by the hopping charge transfer over the nearest acceptor sites in conditions of strong magnetic field. A detailed analysis of the magnetoresistance using the microscopic data obtained from investigations of the resistivity of p$\mathrm{CdSb}: \mathrm{Ni}$ in zero and weak fields [7], demonstrates numerical agreement of the results with predictions of the percolation theory of the nearest-neighbor hopping conductivity in strong magnetic field [10], if the anisotropy of the acceptor states, as well as the explicit dependence of the localization radius on the magnetic field, are taken into account. 


\section{References}

1. Žutić I, Fabian J and Das Sarma S 2004 Rev. Mod. Phys. 76323

2. Laiho R, Lashkul A V, Lisunov K G, Lähderanta E, Ojala I and Zakhvalinskii V S 2006 Semicond. Sci. Technol. 21228

3. Arushanov E K 1986 Prog. Crystal Growth Charact. 131

4. Arushanov E K, Lashkul A V, Pruglo V I, Radautsan S I and Sologub V V 1982 Sov. Phys.Doklady 273

5. Arushanov E K, Lashkul A V, Lisunov K G, Parfen'ev R V and Radautsan S I 1987 Sov. Phys. Solid State 291450

6. Laiho R, Lashkul A V, Lisunov K G, Lähderanta E, Safonchik M O and Shakhov M A 2004 J. Phys.: Condens. Matter 16333

7. Laiho R, Lashkul A V, Lisunov K G, Lähderanta E, Shakhov M A and Zakhvalinskii V S 2008 J. Phys.: Condens. Matter 20295204

8. Ansel'm A I 1978 Introduction to the Theory of Semiconductors (Moscow: Nauka); Seeger K 1985 Semiconductor Physics (Springer Series in Solid-State Science vol 40) (Berlin: Springer)

9. Laiho R, Lashkul A V, Lisunov K G, Lähderanta E, Safonchik M O and Shakhov M A 2004 Semicond. Sci. Technol. 19602

10. Shklovskii B I and Efros A L 1984 Electronic Properties of Doped Semiconductors (Berlin: Springer-Verlag)

11. Mott N F and Davies E A 1979 Electron Processes in Non-Crystalline Materials (Oxford: Clarendon

12. Shklovskii B I 1983 Sov. Phys. - Semicond. 171311

13. Shklovskii B I and Spivak B Z 1991 in Hopping Transport in Solids, edited by Pollak M and Shklovskii B I $\Sigma$ Amsterdam: North-Holland) 271

14. Raikh M E and Wessels G F 1993 Phys. Rev. B 4715609 
TABLE I. The values of the parameters $N_{\mathrm{A}}, a, p_{\mathrm{j}}$ and $E_{\mathrm{A}}$ determined from analysis of the resistivity in zero and weak field [7], constant $(\beta)$ of the high-field dependence of $E_{\mathrm{A}}$ on $B$, the experimental $\left(\left.\eta^{(e x)}\right|_{\varphi=1},\left.\eta^{(e x)}\right|_{\varphi=\varphi_{0}}\right.$ and $\left.\eta^{(e x)}\right)$ and calculated $\left(\eta_{\mathrm{j}}^{(\mathrm{cal})}\right)$ coefficients of the high-field dependence of MR at different orientations of the magnetic field $(j)$.

\begin{tabular}{|c|c|c|c|c|c|c|c|c|c|c|}
\hline $\begin{array}{c}\text { Sample } \\
\text { No. }\end{array}$ & $\begin{array}{c}N_{\mathrm{A}} \\
\left(10^{16} \mathrm{~cm}^{-3}\right)\end{array}$ & $\begin{array}{c}p_{\mathrm{j}} \\
(\AA)\end{array}$ & $\begin{array}{c}E_{\mathrm{A}} \\
(\mathrm{meV})\end{array}$ & $\begin{array}{c}\beta \\
\left(\mathrm{meV} \mathrm{T}^{-1 / 3}\right)\end{array}$ & $\begin{array}{c}\left.\eta^{(e x)}\right|_{\varphi=1} \\
\left(\mathrm{~T}^{-1 / 2}\right)\end{array}$ & $\begin{array}{c}\left.\eta^{(e x)}\right|_{\varphi=\varphi_{0}} \\
\left(\mathrm{~T}^{-1 / 2}\right)\end{array}$ & $\begin{array}{c}\eta^{(e x)} \\
\left(\mathrm{T}^{-1 / 2}\right)\end{array}$ & $\begin{array}{c}\eta_{j}^{(c a l)} \\
\left(\mathrm{T}^{-1 / 2}\right)\end{array}$ & $j$ \\
\hline 1 & 3.61 & 196 & 0.839 & 2.45 & 1.64 & 2.11 & 1.65 & 1.36 & 1.23 & 3 \\
\hline 2 & 3.37 & 180 & 1.008 & 2.50 & 1.53 & 2.09 & 1.69 & 1.50 & 1.46 & 1 \\
\hline 3 & 2.51 & 139 & 1.182 & 2.85 & 1.19 & 2.20 & 1.96 & 2.00 & 2.09 & 2 \\
\hline
\end{tabular}




\section{Figure captions}

Figure 1. Temperature dependence of the resistivity of $\mathrm{p}-\mathrm{CdSb}: \mathrm{Ni}$ at $B=0$. Inset: plots of $\ln \rho$ vs. $T$

${ }^{-1}$ at $B=0$. For convenience the data for $\# 2$ and $\# 3$ are shifted by +0.5 and +2.5 units along the vertical axis. The straight lines are linear fits.

Figure 2. The dependence of the resistivity of p-CdSb:Ni on magnetic field.

Figure 3. The dependence of $\ln \rho$ on $T^{-1}$ for $\# 1$ at $B=11 \mathrm{~T}, 13 \mathrm{~T}$ and $15 \mathrm{~T}$ (from down to up). Inset: plots of $E_{\mathrm{A}}$ vs. $B^{1 / 3}$. The dotted lines are guides for the eye and the solid lines are linear fits.

Figure 4. Plots of $\ln \rho$ vs. $(B \varphi)^{1 / 2}$. The lines are linear fits. 
Fig. 1

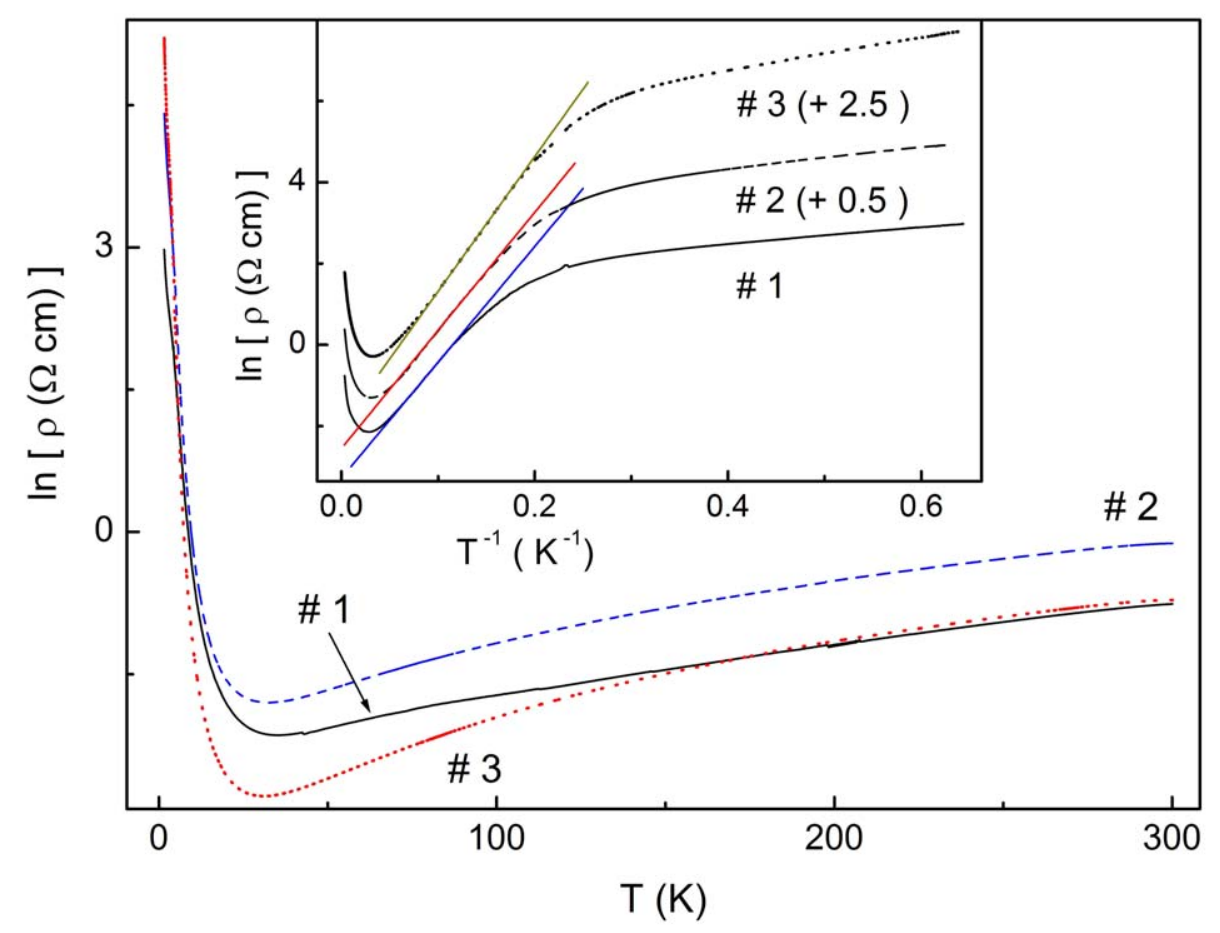


Fig. 2

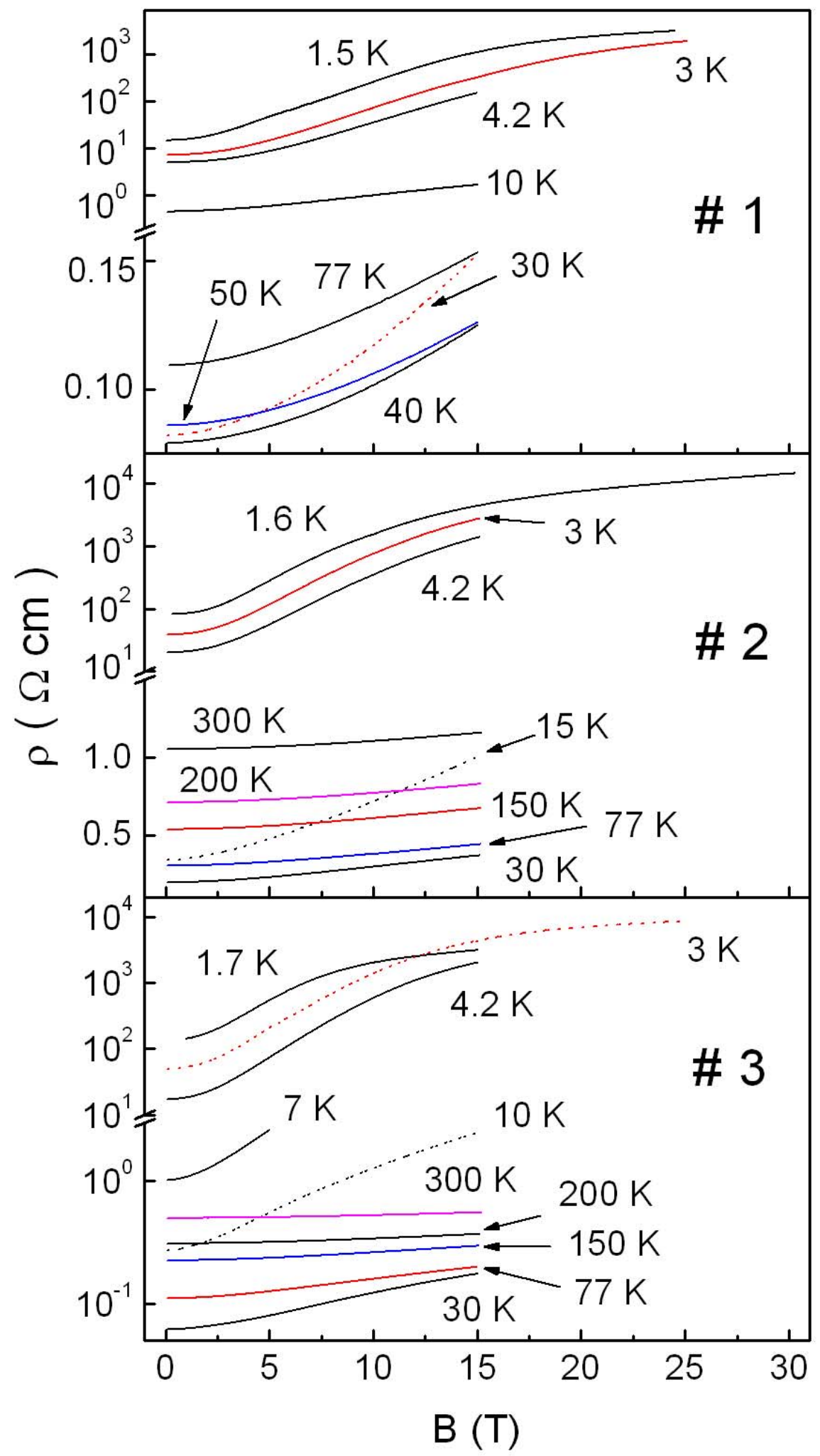


Fig. 3

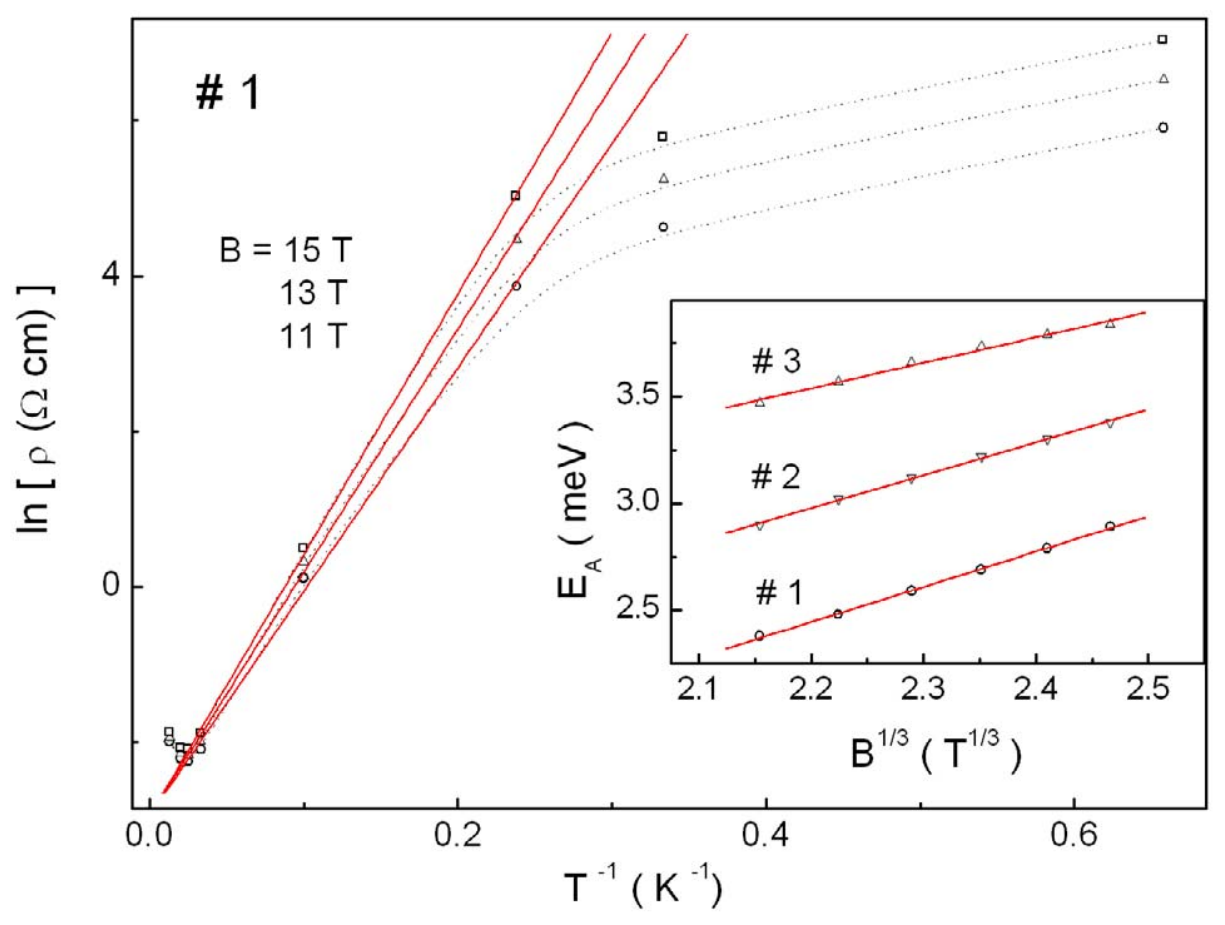


Fig. 4

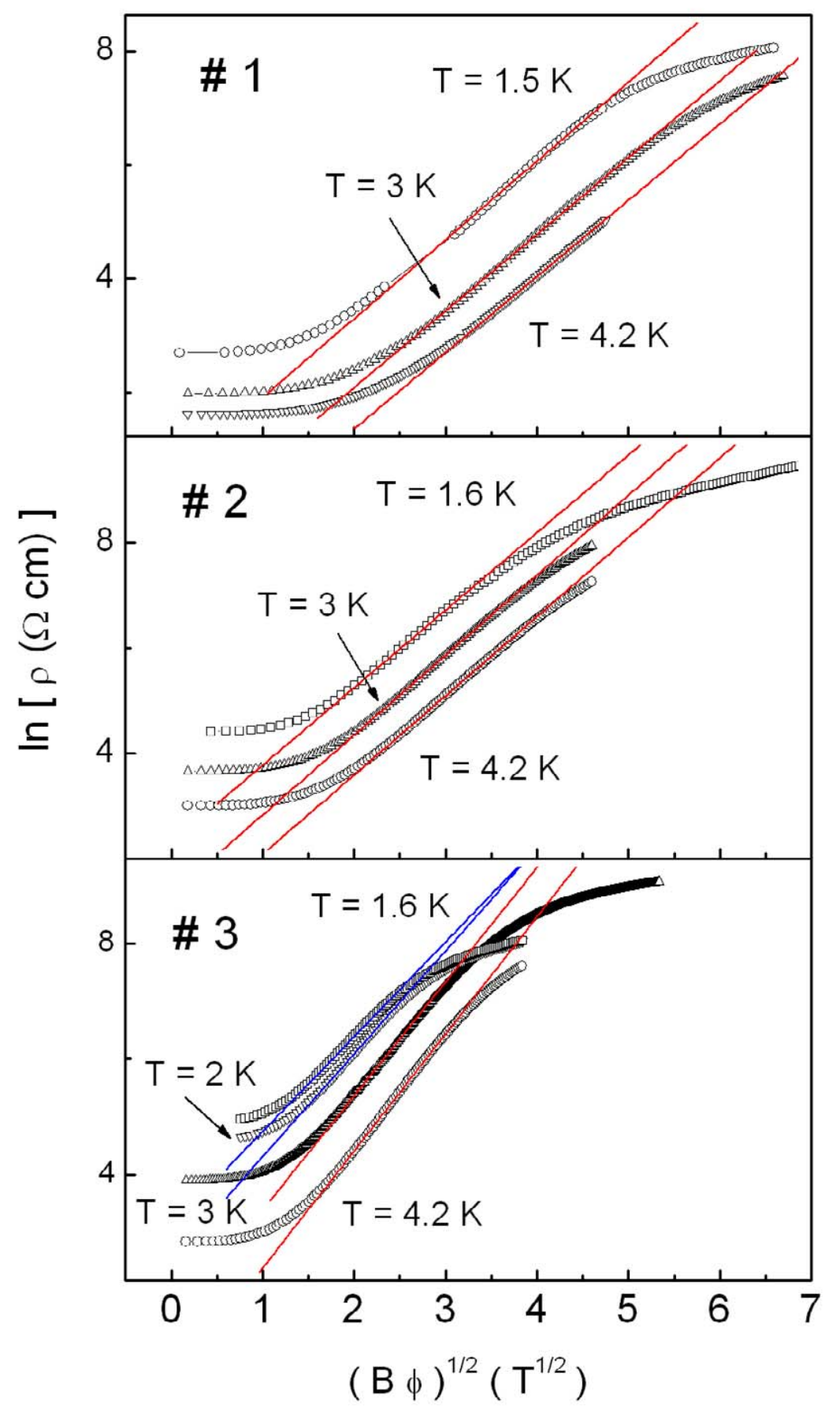

